PROCEEDINGS OF THE

AMERICAN MATHEMATICAL SOCIETY

Volume 130, Number 5, Pages 1323-1332

S 0002-9939(01)06218-9

Article electronically published on October 25, 2001

\title{
SIMPLE ALGEBRAS OF WEYL TYPE, II
}

\author{
KAIMING ZHAO
}

(Communicated by Lance W. Small)

\begin{abstract}
Over a field $\mathbb{F}$ of any characteristic, for a commutative associative algebra $A$, and for a commutative subalgebra $D$ of $\operatorname{Der}_{\mathbb{F}}(A)$, the vector space $A[D]$ which consists of polynomials of elements in $D$ with coefficients in $A$ and which is regarded as operators on $A$ forms naturally an associative algebra. It is proved that, as an associative algebra, $A[D]$ is simple if and only if $A$ is $D$ simple. Suppose $A$ is $D$-simple. Then, (a) $A[D]$ is a free left $A$-module; (b) as a Lie algebra, the subquotient $[A[D], A[D]] /(Z(A[D]) \cap[A[D], A[D]])$ is simple (except for one case), where $Z(A[D])$ is the center of $A[D]$. The structure of this subquotient is explicitly described. This extends the results obtained by $\mathrm{Su}$ and Zhao.
\end{abstract}

\section{INTRODUCTION}

There are many known classes of infinite dimensional simple Lie algebras and simple associative algebras. Generalizations of the infinite dimensional simple Lie algebras of Cartan type over a field of characteristic zero have been obtained by Kawamoto [K], Osborn [O, Dokovic and Zhao [DZ1, DZ2, DZ3, Osborn and Zhao OZ1, OZ2, Zhao [Z3] and Xu [X]. Passman [P] and Jordan [J1] studied the Lie algebras $A D=A \otimes D$ of generalized Witt type constructed from a commutative associative algebra $A$ with an identity element and its commutative derivation subalgebra $D$ over a field $\mathbb{F}$ of arbitrary characteristic. Passman proved that $A D$ is simple if and only if $A$ is $D$-simple (that is, $A$ does not have nontrivial $D$-stable ideals) and $A^{D} D$ acts faithfully on $A$ except for the case char $\mathbb{F}=2, \operatorname{dim}_{\mathbb{F}_{1}} \mathbb{F}_{1} D=1$ and $D(A) \neq A$, where $\mathbb{F}_{1}=A^{D}=\{x \in A \mid D(x)=0\}$.

In [SZ1], for a commutative associative algebra $A$ with an identity element over $\mathbb{F}$ and for the polynomial algebra $\mathbb{F}[D]$ of its commutative derivation subalgebra $D$, $\mathrm{Su}$ and Zhao constructed the associative algebras $A \otimes \mathbb{F}[D]$ of Weyl type, and proved that $A \otimes \mathbb{F}[D]$ is simple if and only if $A$ is $D$-simple and $A^{D} \otimes \mathbb{F}[D]$ acts faithfully on $A$, both as a Lie algebra (modulo its center) and as an associative algebra. The simple algebras $A \otimes \mathbb{F}[D]$ (or modulo its center as Lie algebras) are always infinite dimensional. But as operators on $A$, some nonzero elements in $A \otimes \mathbb{F}[D]$ are possibly zero operators on $A$. In this case, $A \otimes \mathbb{F}[D]$ certainly cannot be a simple Lie algebra (modulo its center) or a simple associative algebra. If we directly consider such

Received by the editors August 28, 2000 and, in revised form, November 20, 2000.

2000 Mathematics Subject Classification. Primary 16W10, 16W25, 17B20, 17B65, 17B05, $17 \mathrm{~B} 68$.

Key words and phrases. Simple Lie algebra, simple associative algebra, derivation.

This work was supported by the Hundred Talents Program of Chinese Academy of Sciences and by NSF of China. 
operators on $A$, we can avoid this case so that we still get simple algebras. This is the objective of this paper.

Throughout this paper, $\mathbb{F}$ is a field of arbitrary characteristic.

In this paper, for a commutative associative algebra $A$ with an identity element over $\mathbb{F}$ and for a commutative derivation subalgebra $D \subset \operatorname{Der}(A)$, we study the associative algebra $A[D]$ which consists of polynomials of elements in $D$ with coefficients in $A$ and which is regarded as operators on $A$. These associative algebras can also be realized as iterated skew polynomial rings if $\operatorname{dim} D<\infty$ (see [J2]). As an associative algebra, it is proved that $A[D]$ is simple if and only if $A$ is $D$-simple (see Theorem 2.3). Suppose $A$ is $D$-simple, then (a) $A[D]$ is a free left $A$-module (see Theorem [3.5); (b) as a Lie algebra, the subquotient $[A[D], A[D]] /(Z(A[D]) \cap[A[D], A[D]])$ is simple (except for one case), where $Z(A[D])$ is the center of $A[D]$. The structure of this subquotient is explicitly described (see Theorems 3.8 3.9). The Lie algebras obtained by Su and Zhao in [SZ1] are some infinite dimensional Lie algebra studied in this paper. In particular we can get a lot of finite dimensional simple modular Lie algebras. At the end of this paper, we give some finite or infinite dimensional examples which cannot be covered by [SZ1].

\section{Simple associative algebras of Weyl type}

Let $A$ be a commutative associative algebra with an identity element 1 over $\mathbb{F}$, and let $D$ be a nonzero $\mathbb{F}$-vector space spanned by some commuting $\mathbb{F}$-derivations of $A$. Let

$$
\left\{\partial_{i} \mid i \in \dot{I}\right\}, \quad \text { where } \dot{I} \text { is some indexing set, }
$$

be an $\mathbb{F}$-basis of $D$. Denote

$$
\begin{array}{r}
\dot{J}=\left\{\alpha=\left(\alpha_{i} \mid i \in \dot{I}\right) \mid \alpha_{i} \in \mathbb{Z}_{+}, \forall i \in \dot{I} \text { and } \alpha_{i}=0\right. \\
\text { for all but a finite number of } i \in \dot{I}\},
\end{array}
$$

where $\alpha=\left(\alpha_{i} \mid i \in \dot{I}\right)$, simply denoted by $\alpha=\left(\alpha_{i}\right)$, is a collection of nonnegative integers indexed by $\dot{I}$. Define

$$
|\alpha|=\sum_{i \in \dot{I}} \alpha_{i}
$$

which is called the level of $\alpha$. For any $\alpha \in \dot{J}, x \in A$, we define the operator on $A$

$$
x \partial^{\alpha}=x \prod_{i \in \dot{I}} \partial_{i}^{\alpha_{i}}
$$

by $\left(x \partial_{1} \partial_{2} \cdots \partial_{n}\right)(y)=x\left(\partial_{1}\left(\partial_{2} \cdots\left(\partial_{n}(y)\right) \cdots\right)\right)$ for any $y \in A$. Let $A[D]$ be the $\mathbb{F}$-vector space spanned by all the following operators on $A$ :

$$
x \partial^{\alpha}, \quad \forall x \in A, \alpha \in \dot{J} .
$$

If we define the product of $A[D]$ as the composition of operators on $A$, then $A[D]$ becomes an associative algebra. It is not difficult to verify that

$$
\left(u \partial^{\alpha}\right) \cdot\left(v \partial^{\beta}\right)=u \sum_{\gamma \in j(\alpha)}\left(\begin{array}{l}
\alpha \\
\gamma
\end{array}\right) \partial^{\gamma}(v) \partial^{\alpha+\beta-\gamma},
$$


for all $u, v \in A, \alpha, \beta \in \dot{J}$, where

$$
\begin{aligned}
& \dot{J}(\alpha)=\left\{\gamma \in \dot{J} \mid \gamma_{i} \leq \alpha_{i}, \forall i \in \dot{I}\right\} \\
& \left(\begin{array}{l}
\alpha \\
\gamma
\end{array}\right)=\prod_{i \in \dot{I}}\left(\begin{array}{c}
\alpha_{i} \\
\gamma_{i}
\end{array}\right), \quad \gamma \in \dot{J}(\alpha) .
\end{aligned}
$$

We call the associative algebra $A[D]$ an associative algebra of Weyl type. This is different from that in [SZ1].

Remark. It is clear that $A$ is a left $A[D]$-module and $A[D]$ is a left $A$-module.

For any

$$
x=\sum_{\alpha \in J} u_{\alpha} \partial^{\alpha} \in A[D],
$$

the expression in (2.8) is sometimes not unique. An expression of $x$ in (2.8) is called principal if the integer $\max \left\{|\alpha| \mid a_{\alpha} \neq 0\right\}$ is minimal. We denote this integer by $h(x)$. Set

$$
\mathbb{F}_{1}=A^{D}=\{u \in A \mid D(u)=0\} .
$$

We use this notation because it is a field extension of $\mathbb{F}$ when $A$ is $D$-simple, i.e., $A$ has no nontrivial $D$-stable ideals; see [J1].

Lemma 2.1. $A \cap A[D] D=0$.

Proof. Suppose $x \in A \cap A[D] D$. Since $x \in A$, then the action of $x$ on 1 is $x \cdot 1=x$. On the other hand, since $x \in A[D] D$ and $D(1)=0$, then the action of $x$ on 1 is 0 . So $x=0$. Thus $A \cap A[D] D=0$.

Lemma 2.2. The center $Z(A[D])$ of $A[D]$ is $\mathbb{F}_{1}$.

Proof. It is clear that $\mathbb{F}_{1} \subset Z(A[D])$.

Suppose $x \in Z(A[D])$. If $h(x)>0$, write $x=X_{0}+X_{1}$ where $X_{0} \in A$ and $X_{1} \in A[D] D$. Then $X_{1} \neq 0$. Choose $a \in A$ such that $X_{0}(a) \neq 0$. It follows that

$$
0=[X, a]=\left[X_{1}, a\right]=X_{1}(a)+Y,
$$

where $Y \in A[D] D$. From Lemma 2.1] we deduce that $X_{1}(a)=Y=0$, a contradiction. So $h(x)=0$. We have

$$
x \partial=\partial x=x \partial+\partial(x), \forall \partial \in D,
$$

to give $\partial(x)=0$ for all $\partial \in D$, i.e., $x \in \mathbb{F}_{1}$. Therefore Lemma 2.2 follows.

Theorem 2.3. The associative algebra $A[D]$ is simple if and only if $A$ is $D$-simple.

Proof. " $\Rightarrow$ ": Suppose $A$ is not $D$-simple. Choose a nonzero proper $D$-ideal $\mathcal{I}$. Then clearly $\mathcal{I}[D]$ is a nonzero ideal of $A[D]$. Since $A[D]$ is simple, then $\mathcal{I}[D]=A[D]$, in particular, $A \subset \mathcal{I}[D]$. From Lemma 2.1 we know that $A \subset \mathcal{I}$, a contradiction. Thus $A$ is $D$-simple. 
" $\Leftarrow$ ": Suppose $L$ is a nonzero ideal of $A[D]$. It suffices to show that $L=A[D]$.

Claim 1. $L \cap A \neq 0$.

Suppose that $L \cap A=0$. Choose $X \in L \backslash\{0\}$ such that $h(X)$ is minimal. So $h(X)>0$. Write $X=X_{0}+X_{1}$ where $X_{0} \in A$ and $X_{1} \in A[D] D$. Then $X_{1} \neq 0$. Choose $a \in A$ such that $X_{0}(a) \neq 0$. It follows that

$$
X^{\prime}=[X, a]=\left[X_{1}, a\right]=X_{1}(a)+Y \in L,
$$

where $Y \in A[D] D$. From the computation (2.6) we deduce that $h\left(X^{\prime}\right)=h(Y)<$ $h(X)$. By the minimality of $h(X)$ we deduce that $X^{\prime}=0$. Applying Lemma 2.1 gives $X_{1}(a)=Y=0$, a contradiction. Thus Claim 1 follows.

It is clear that $L \cap A$ is a $D$-ideal of $A$. Since $A$ is $D$-simple, then $L \cap A=A$. In particular $1 \in L$. Therefore $L=A[D]$.

\section{Simple Lie algebras of Weyl type}

Let $A$ be a commutative associative algebra with an identity element 1 over $\mathbb{F}$, and let $D$ be a nonzero $\mathbb{F}$-vector space spanned by some commuting $\mathbb{F}$-derivations of $A$ such that $A$ is $D$-simple. It is well known that $\mathbb{F}_{1}$ is a field extension of $\mathbb{F}$. From Theorem 2.3 we know that the associative algebra $A[D]$ is simple.

We denote the induced Lie algebra from the associative algebra $A[D]$ by $A[D]^{L}$.

In this section we shall first study the structure of $A[D]$ as a left $A$-module, then investigate the Lie structure of $A[D]$. We still use the notations in (2.1) and (2.2).

The following lemma will be often used.

Lemma 3.1. (a) If $x \partial^{\alpha}=0$ for some $x \in A \backslash\{0\}, \alpha \in J$, then $\partial^{\alpha}=0$.

(b) $A \subset\left[A[D]^{L}, A[D]^{L}\right]$.

Proof. (a) Let $C=\left\{x \in A \mid x \partial^{\alpha}=0\right\}$. It is easy to verify that $C$ is a nonzero $D$-ideal of $A$. Since $A$ is $D$-simple, then $C=A$. It yields $\partial^{\alpha}=0$.

(b) From $[x \partial, y]=x \partial(y) \in\left[A[D]^{L}, A[D]^{L}\right]$ for all $x, y \in A$ and $\partial \in D$, we know that $A D(A) \subset\left[A[D]^{L}, A[D]^{L}\right]$. Since $A D(A)$ is a nonzero $D$-ideal of $A$ and $A$ is $D$-simple, then $A=A D(A)$. Thus $A \subset\left[A[D]^{L}, A[D]^{L}\right]$.

If $\operatorname{char} \mathbb{F}=p>0$, observe that for any $\partial \in \operatorname{Der}_{\mathbb{F}}(A)$, one has $\partial^{p} \in \operatorname{Der}_{\mathbb{F}}(A)$. Let

$$
\mathcal{D}=\operatorname{Der}_{\mathbb{F}}(A) \cap \mathbb{F}_{1}[D],
$$

where $\mathbb{F}_{1}[D]$ is the polynomial algebra of elements in $D$, and let

$$
\left\{d_{i} \mid i \in I\right\}
$$

be an $\mathbb{F}_{1}$ basis for $\mathcal{D}$. It is clear that $D \subset \mathcal{D} . J=\left\{\alpha=\left(\alpha_{i} \mid i \in I\right) \mid \alpha_{i} \in \mathbb{Z}_{+}, \forall i \in I\right.$ and $\alpha_{i}=0$ for all but a finite number of $\left.i \in I\right\}$, where $\alpha=\left(\alpha_{i} \mid i \in I\right)$, and let

$$
\begin{aligned}
& J_{0}=\left\{\alpha=\left(\alpha_{i} \mid i \in I\right) \mid 0 \leq \alpha_{i} \in \leq p-1, \forall i \in I\right. \\
&\text { and } \left.\alpha_{i}=0 \text { for all but a finite number of } i \in I\right\},
\end{aligned}
$$

where $\alpha=\left(\alpha_{i} \mid i \in I\right)$ will be simply denoted by $\alpha=\left(\alpha_{i}\right)$. We also use the notation $d^{\alpha}=\prod_{i \in I} d^{\alpha_{i}}, \forall \alpha \in J_{0}$. If $\operatorname{char} \mathbb{F}=p$, then

$$
A[D]=\sum_{\alpha \in J_{0}} A d^{\alpha} .
$$

If $\operatorname{char} \mathbb{F}=0$, we still have (3.4) by replacing $J_{0}$ with $J$. 
Lemma 3.2. (a) Suppose char $\mathbb{F}=p>0$ and $J_{1} \subset J_{0}$. Then the sum $\sum_{\alpha \in J_{1}} A d^{\alpha}$ is direct if and only if the sum $\sum_{\alpha \in J_{1}} \mathbb{F}_{1} d^{\alpha}$ is direct.

(b) Suppose char $\mathbb{F}=0$ and $J_{1} \subset J$. Then the sum $\sum_{\alpha \in J_{1}} A d^{\alpha}$ is direct if and only if the sum $\sum_{\alpha \in J_{1}} \mathbb{F}_{1} d^{\alpha}$ is direct.

Proof. (a) " $\Rightarrow$ ": This direction is clear.

" $\Leftarrow$ ": Suppose $\sum_{\alpha \in J_{1}} A d^{\alpha}$ is not direct. There exists $J_{2}=\left\{\alpha^{(0)}, \alpha^{(2)}, \ldots, \alpha^{(r)}\right\}$ $\subset J_{1}$ such that $\left|J_{2}\right|>1$ and $A d^{\alpha^{(0)}} \cap \sum_{i=1}^{r} A d^{\alpha^{(i)}} \neq 0$. Choose such a minimal $r$. Let

$$
a d^{\alpha^{(0)}}=\sum_{i=1}^{r} a_{i} d^{\alpha^{(i)}} \in\left(A d^{\alpha^{(0)}} \cap \sum_{i=1}^{r} A d^{\alpha^{(i)}}\right) \backslash\{0\}
$$

for some $a \in A \backslash\{0\}, a_{i} \in A$, and let $I_{1}=\{0,1, \ldots, r\}$. It follows that $d^{\alpha^{(i)}} \neq 0$ for any $i \in I_{1}$. For any $0 \leq j \leq r$, let

$$
B_{j}=\left\{x \in A \mid x d^{\alpha^{(j)}} \in \sum_{i \in I_{1} \backslash\{j\}} A d^{\alpha^{(i)}}\right\} .
$$

Then it is easy to see that $B_{j}$ is a nonzero $D$-ideal of $A$. Since $A$ is $D$-simple, it follows that $B_{j}=A$ for any $j \in I_{1}$. Then

$$
d^{\alpha^{(0)}}=\sum_{i \in I_{1} \backslash\{0\}} a_{i} d^{\alpha^{(i)}}, \quad a_{i} \in A .
$$

By taking brackets with $\partial \in \mathcal{D}$, we deduce that

$$
0=\left[\partial, d^{\alpha^{(0)}}\right]=\left[\partial, \sum_{i \in I_{1} \backslash\{0\}} a_{i} d^{\alpha^{(i)}}\right]=\sum_{i \in I_{1} \backslash\{0\}} \partial\left(a_{i}\right) d^{\alpha^{(i)}} .
$$

From the minimality of $r$, we must have

$$
\partial\left(a_{i}\right) d^{\alpha^{(i)}}=0, \quad \forall \partial \in \mathcal{D}, 1 \leq i \leq r .
$$

Suppose $D\left(a_{i}\right) \neq 0$ for some $i \in I_{1}$, say $D\left(a_{1}\right) \neq 0$. Lemma 3.1 yields $d^{\alpha^{(1)}}=0$, a contradiction. Thus $D\left(a_{i}\right)=0$ for all $i \in I_{1}$, i.e., $a_{i} \in \mathbb{F}_{1}$ in (3.5). Therefore the $\operatorname{sum} \sum_{\alpha \in J_{1}} \mathbb{F}_{1} d^{\alpha}$ is not direct. This direction of (a) follows.

The proof for part (b) is similar to that of (a).

Lemma 3.3. (a) If char $\mathbb{F}=p>0$, then $A[D]=\bigoplus_{\alpha \in J_{0}} A d^{\alpha}$.

(b) If $\operatorname{char} \mathbb{F}=0$, then $A[D]=\bigoplus_{\alpha \in J} A d^{\alpha}$.

Proof. (a) From (3.4), we know that it suffices to show the sum in (3.4) is direct.

Suppose the sum in (3.4) is not direct. From Lemmas 2.1] 3.1, there exists $J_{2}=\left\{\alpha^{(0)}, \alpha^{(2)}, \ldots, \alpha^{(r)}\right\} \subset J_{0} \backslash\{0\}$ such that $\mathbb{F}_{1} d^{\alpha^{(0)}} \cap \sum_{i=1}^{r} \mathbb{F}_{1} d^{\alpha^{(i)}} \neq 0$. Let $I_{1}=\{0,1, \ldots, r\}$ and $h\left(J_{2}\right)=\max \left\{\left|\alpha^{(i)}\right| \mid i \in I_{1}\right\}$. Then $h\left(J_{2}\right)>1$. Choose such a $J_{2}$ such that $h\left(J_{2}\right)$ is minimal, and then $r$ is minimal. Let

$$
d^{\alpha^{(0)}}=\sum_{i=1}^{r} a_{i} d^{\alpha^{(i)}} \in\left(\mathbb{F}_{1} d^{\alpha^{(0)}} \cap \sum_{i=1}^{r} \mathbb{F}_{1} d^{\alpha^{(i)}}\right) \backslash\{0\}
$$


for some $a_{i} \in \mathbb{F}_{1}, 1 \leq i \leq r$. Denote $a_{0}=1$. It follows that $a_{i} d^{\alpha^{(i)}} \neq 0$ for any $i \in I_{1}$. Let

$$
J_{0}(\alpha)=\left\{\beta \in J_{0} \backslash\{0, \alpha\} \mid \beta_{i} \leq \alpha_{i}, \forall i \in I\right\} .
$$

Rewrite (3.7) to give

$$
\sum_{i=0}^{r} a_{i} d^{\alpha^{(i)}}=0
$$

For any $x \in A$, it is clear that $\sum_{i=0}^{r} a_{i} d^{\alpha^{(i)}}(x)=0$; then

$$
\begin{aligned}
0 & =\left[\sum_{i=0}^{r} a_{i} d^{\alpha^{(i)}}, x\right]=\sum_{i=0}^{r} \sum_{\gamma \in J_{0}\left(\alpha^{(i)}\right)} a_{i}\left(\begin{array}{c}
\alpha^{(i)} \\
\gamma
\end{array}\right) d^{\alpha^{(i)}-\gamma}(x) d^{\gamma} \\
& =\sum_{\gamma \in J_{0}, 0<|\gamma|<h\left(J_{2}\right)}\left(\sum_{i=0}^{r} a_{i}\left(\begin{array}{c}
\alpha^{(i)} \\
\gamma
\end{array}\right) d^{\alpha^{(i)}-\gamma}(x)\right) d^{\gamma} .
\end{aligned}
$$

From the minimality of $h\left(J_{2}\right)$, it follows that

$$
\sum_{i=0}^{r} a_{i}\left(\begin{array}{c}
\alpha^{(i)} \\
\gamma
\end{array}\right) d^{\alpha^{(i)}-\gamma}(x) d^{\gamma}=0
$$

for all $x \in A$, and all $\gamma \in J$ with $0<h(\gamma)<h\left(J_{2}\right)$. Since $d^{\gamma} \neq 0$, from Lemma 3.1 we see that

$$
\sum_{i=0}^{r} a_{i}\left(\begin{array}{c}
\alpha^{(i)} \\
\gamma
\end{array}\right) d^{\alpha^{(i)}-\gamma}(x)=0
$$

for all $x \in A$, and all $\gamma \in J$ with $0<h(\gamma)<h\left(J_{2}\right)$. So

$$
\sum_{i=0}^{r} a_{i}\left(\begin{array}{c}
\alpha^{(i)} \\
\gamma
\end{array}\right) d^{\alpha^{(i)}-\gamma}=0
$$

for all $\gamma \in J$ with $0<h(\gamma)<h\left(J_{2}\right)$. Since $\alpha_{j}^{(i)} \leq p-1$, then $a_{i}\left(\begin{array}{c}\alpha^{(i)} \\ \gamma\end{array}\right) \neq 0$ if $\gamma \in$ $J_{0}\left(\alpha^{(i)}\right)$. This contradicts the minimality of $h\left(J_{2}\right)$. Therefore $A[D]=\bigoplus_{\alpha \in J_{0}} A d^{\alpha}$. Part (a) is true.

The proof of part (b) is similar to that of part (a).

Lemma 3.4. (a) If char $\mathbb{F}=p>0$, then $d^{\alpha} \neq 0$, for any $\alpha \in J_{0}$.

(b) If char $\mathbb{F}=0$, then $d^{\alpha} \neq 0$, for any $\alpha \in J$.

Proof. (a) It is obvious that $d^{\alpha} \neq 0$ for any $\alpha \in J_{0}$ with $|\alpha|<2$.

Suppose $d^{\alpha}=0$ for some $\alpha \in J_{0}$ with $|\alpha| \geq 2$. Choose an $\alpha$ such that $|\alpha|$ is minimal. For any $x \in A$, we see $d^{\alpha}(x)=0$. Then

$$
0=\left[d^{\alpha}, x\right]=\sum_{\beta \in J_{0}(\alpha)}\left(\begin{array}{l}
\alpha \\
\beta
\end{array}\right) d^{\alpha-\beta}(x) d^{\beta}=0, \quad \forall x \in A,
$$

where $J_{0}(\alpha)$ is as in (3.8). Applying Lemma 3.3 and noting that $\left(\begin{array}{l}\alpha \\ \beta\end{array}\right) \neq 0$ for any $\beta \in J_{0}(\alpha)$, since $|\alpha|$ is minimal we get $d^{\alpha-\beta}(x) d^{\beta}=0, \forall x \in A, \beta \in J_{0}(\alpha)$. Applying Lemma 3.1 and noting that $d^{\beta} \neq 0$ for any $\beta \in J_{0}(\alpha)$, we obtain $d^{\alpha-\beta}(x)=0$, $\forall x \in A$, i.e., $d^{\alpha-\beta} \neq 0$ for any $\beta \in J_{0}(\alpha)$. This contradicts the minimality of $|\alpha|$. Therefore (a) follows.

The proof of part (b) is similar to that of part (a). 
Theorem 3.5. Suppose $A$ is $D$-simple. Then $A[D]$ is a free A-module. Furthermore,

(a) if char $\mathbb{F}=p>0$, then $A[D]$ is a free A-module with a basis $\left\{d^{\alpha} \mid \alpha \in J_{0}\right\}$;

(b) if char $\mathbb{F}=0$, then $A[D]$ is a free A-module with a basis $\left\{d^{\alpha} \mid \alpha \in J\right\}$.

Proof. This theorem directly follows from Lemmas 3.2 , 3.3 and 3.4 .

Lemma 3.6. (a) Suppose that $\operatorname{char} \mathbb{F}=p>0$, and that $\left\{d_{i} \mid 1 \leq i \leq n\right\} \subset \mathcal{D}$ are $\mathbb{F}_{1}$ linearly independent. Then, for any integers $0 \leq r_{1}, r_{2}, \ldots, r_{n} \leq p-1$ with $r_{1}+r_{2}+\cdots+r_{n} \leq n(p-1)-1$, we have

$$
A d_{1}^{r_{1}} d_{2}^{r_{2}} \cdots d_{n}^{r_{n}} \subset\left[A[D]^{L}, A[D]^{L}\right] .
$$

(b) Suppose that char $\mathbb{F}=0$, and that $\left\{d_{i} \mid 1 \leq i \leq n\right\} \subset \mathbb{F}_{1} D$ are $\mathbb{F}_{1}$ linearly independent. Then, for any integers $r_{1}, r_{2}, \ldots, r_{n} \geq 0$, we have

$$
A d_{1}^{r_{1}} d_{2}^{r_{2}} \cdots d_{n}^{r_{n}} \subset\left[A[D]^{L}, A[D]^{L}\right] .
$$

Proof. (a) In this proof, we define $J_{0}=\left\{\left(r_{1}, r_{2}, \ldots, r_{n}\right) \mid 0 \leq r_{1}, r_{2}, \ldots, r_{n} \leq p-1\right\}$. Let $\beta=(p-1, p-1, \ldots, p-1) \in J_{0}, J_{1}=J_{0} \backslash\{0\}$ and $J_{2}=J_{1} \backslash\{\beta\}$. Denote $W=\left[A[D]^{L}, A[D]^{L}\right]$. For any $x, y \in A$, we deduce

$$
\left[x d_{1}^{p-1} d_{2}^{p-1} \cdots d_{n}^{p-1}, y\right]=\sum_{\alpha \in J_{1}}\left(\begin{array}{l}
\beta \\
\alpha
\end{array}\right) x d^{\alpha}(y) d^{\beta-\alpha} \in W .
$$

By using Lemma 3.1 we obtain that

$$
\sum_{\alpha \in J_{2}}\left(\begin{array}{l}
\beta \\
\alpha
\end{array}\right) x d^{\alpha}(y) d^{\beta-\alpha} \in W, \quad \forall x, y \in A .
$$

Note that the coefficients $\left(\begin{array}{c}\beta \\ \alpha\end{array}\right)$ are not zero. Since $\left\{d^{\alpha} \mid \alpha \in J_{2}\right\}$ is $A$ linearly independent (Theorem 3.5), there exist $a_{\alpha} \in A$ for all $\alpha \in J_{2}$ such that the $\left(p^{n}-2\right) \times\left(p^{n}-2\right)$ determinant

$$
w=\operatorname{det}\left(d^{\alpha}\left(a_{\gamma}\right)\right)_{\alpha, \gamma \in J_{2}} \in A \backslash\{0\} .
$$

In (3.9), if we take $y$ to be $a_{\gamma}$ respectively, we obtain $p^{n}-2$ equations

$$
x \sum_{\alpha \in J_{2}}\left(\begin{array}{l}
\beta \\
\alpha
\end{array}\right) d^{\alpha}\left(a_{\gamma}\right) d^{\beta-\alpha} \in W, \quad \forall x \in A, \gamma \in J_{2} .
$$

Applying linear algebra knowledge to (3.11), we deduce that $A w d^{\alpha} \in L$ for all $\alpha \in J_{2}$. Let $X_{\alpha}=\left\{x \in A \mid x d^{\alpha} \in L\right\}$. Then $A w \subset X_{\alpha}$, and $D\left(X_{\alpha}\right) \subset X_{\alpha}$. By induction on $|\alpha|$ we can easily show that $A d^{\alpha}(w) \subset X_{\alpha}$. Thus $A[D](w) \subset X_{\alpha}$. Note that $A[D](w)$ is a nonzero $D$-ideal of $A$. Since $A$ is $D$-simple, we must have $A[D](w)=A$, so $X_{\alpha}=A$ for all $\alpha \in J_{2}$. Therefore $A d^{\alpha} \subset L$ for all $\alpha \in J_{2}$. Part (a) follows.

The proof of part (b) is similar to that of part (a).

Theorem 3.7. (a) Suppose that char $\mathbb{F}=p>0$, and that $\left\{d_{i} \mid 1 \leq i \leq n\right\}$ is an $\mathbb{F}_{1}$ basis of $\mathcal{D}$ with $n<\infty$. Let $\beta=(p-1, p-1, \ldots, p-1) \in J_{0}$. Then

$$
W=\left[A[D]^{L}, A[D]^{L}\right]=\bigoplus_{\alpha \in J_{0} \backslash\{\beta\}} A d^{\alpha} \oplus D(A) d^{\beta} .
$$

(b) Suppose that $\operatorname{char} \mathbb{F}=0$, or that $\operatorname{dim} \mathcal{D}=\infty$ if $\operatorname{char} \mathbb{F}=p>0$. Then $A[D]^{L}=\left[A[D]^{L}, A[D]^{L}\right]$. 
Proof. (a) Let $\delta_{i}=\left(\delta_{1, i}, \delta_{2, i}, \ldots, \delta_{n, i}\right) \in J_{0}$. From Lemma 3.6 we know that $\bigoplus_{\alpha \in J_{0} \backslash\{\beta\}} A d^{\alpha} \subset W$. The computation $\left[A d^{\beta}, D\right]=D(A) d^{\beta}$ yields $D(A) d^{\beta} \subset W$. Note that $D(A)=\mathcal{D}(A)$. For any $\alpha \in J_{0} \backslash\{\beta\}$, and any $x, y \in A$, we obtain that

$$
\begin{aligned}
{\left[x d^{\alpha}, y d^{\beta-\alpha+\delta_{i}}\right] } & \equiv\left(x \alpha_{i} d_{i}(y)-y\left(p-\alpha_{i}\right) d_{i}(x)\right) d^{\beta} \quad(\bmod L) \\
& \equiv \alpha_{i} d_{i}(x y) d^{\beta} \quad(\bmod L),
\end{aligned}
$$

where $L=\left[A[D]^{L}, A[D]^{L}\right]$. Thus (3.12) holds.

Part (b) directly follows from Lemma 3.6

Theorem 3.8. Suppose $A$ is D-simple. Then the Lie algebra

$$
\overline{A[D]}=\left[A[D]^{L}, A[D]^{L}\right] /\left(\mathbb{F}_{1} \cap\left[A[D]^{L}, A[D]^{L}\right]\right)
$$

is simple except when char $\mathbb{F}=2, A=\mathbb{F}_{1}[t] /\left(t^{2}-a\right)$ and $A[D]=A \oplus \mathbb{F}_{1}(d / d t) \oplus$ $\mathbb{F}_{1}(t d / d t)$ for some $a \in \mathbb{F}_{1}^{*}$ where $d / d t$ is the ordinary differential.

Proof. From Lemma 3.1 we see that

$$
A \subset\left[A[D]^{L}, A[D]^{L}\right], \quad \overline{A[D]} \neq 0 .
$$

From $[\mathrm{H}]$, we know that the Lie algebra

$$
\overline{A[D]}=\left[A[D]^{L}, A[D]^{L}\right] /\left(\mathbb{F}_{1} \cap\left[A[D]^{L}, A[D]^{L}\right]\right)
$$

is simple except when char $\mathbb{F}=2, \operatorname{dim}_{\mathbb{F}_{1}} A[D]=4$.

Next suppose char $\mathbb{F}=2, \operatorname{dim}_{\mathbb{F}_{1}} A[D]=4$.

From Lemma 2.1 we see that $\operatorname{dim}_{\mathbb{F}_{1}} A=2,3$. If $\operatorname{dim}_{\mathbb{F}_{1}} A=3$, let $A=\mathbb{F}_{1} \oplus$ $\mathbb{F}_{1} x_{1} \oplus \mathbb{F}_{1} x_{2}$. Then $\mathbb{F}_{1} D=\mathbb{F}_{1} \partial$. We must have $A \partial=\mathbb{F}_{1} \partial$. It follows that $\partial(A)=$ $A D(A)=A$, contrary to $\partial\left(\mathbb{F}_{1}\right)=0$. Thus $\operatorname{dim}_{\mathbb{F}_{1}} A=2$.

Let $A=\mathbb{F}_{1} \oplus \mathbb{F}_{1} x$. Let $x^{2}=a x+b$ where $a, b \in \mathbb{F}_{1}$. For any $\partial \in D \backslash\{0\}$, we have $0=\partial\left(x^{2}\right)=a \partial(x)$, to give $a=0$ since $\partial(x) \neq 0$. Thus $x^{2}-b=0$, i.e., $A \simeq \mathbb{F}_{1}(t) /\left(t^{2}-b\right)$. It follows that $\operatorname{Der}_{\mathbb{F}_{1}} A=\mathbb{F}_{1}(d / d t)+\mathbb{F}_{1}(t d / d t)$. If $b=0$, we see that $A$ is not $\operatorname{Der}(A)$-simple. So $b \neq 0$. Then we must have $A[D]=$ $A \oplus \mathbb{F}_{1}(d / d t) \oplus \mathbb{F}_{1}(t d / d t)$.

We call the Lie algebra $\overline{A[D]}$ a Lie algebra of Weyl type.

From Theorem 3.7 and Theorem 3.8 , we have

Theorem 3.9. (a) Suppose $A$ is D-simple. Then $A[D]^{L} /\left(\mathbb{F}_{1} \cap\left[A[D]^{L}, A[D]^{L}\right]\right)$ is a simple Lie algebra if and only if one of the following conditions holds:

(I) $\operatorname{char} \mathbb{F}=0$;

(II) $\operatorname{char} \mathbb{F}=p>0$ and $\operatorname{dim} \mathcal{D}=\infty$;

(III) $\operatorname{char} \mathbb{F}=p>0, \operatorname{dim} \mathcal{D}<\infty$ and $D(A)=A$.

(b) Suppose char $\mathbb{F}=p>0$ and $\operatorname{dim} \mathcal{D}<\infty$. Let $\left\{d_{i} \mid 1 \leq i \leq n\right\}$ be an $\mathbb{F}_{1}$ basis of $\mathcal{D}$ with $n<\infty$. Let $\beta=(p-1, p-1, \ldots, p-1) \in J_{0}$. Then the subquotient $\left(\bigoplus_{\alpha \in J_{0} \backslash\{\beta\}} A d^{\alpha} \oplus D(A) d^{\beta}\right) / \mathbb{F}_{1}$ is a simple Lie algebra except when $\operatorname{char} \mathbb{F}=2, A=$ $\mathbb{F}_{1}[t] /\left(t^{2}-a\right)$ and $A[D]=A \oplus \mathbb{F}_{1}(d / d t) \oplus \mathbb{F}_{1}(t d / d t)$ for some $a \in \mathbb{F}_{1}^{*}$.

Remark. If char $\mathbb{F}=0$, then in Theorem 3.9 we have $A[D]=A \otimes \mathbb{F}[D]$, and $\operatorname{dim} A[D]=\infty$. 
The converse of Theorem $3.9(\mathrm{a})$ is generally not true. See the following example.

Example 3.10. Suppose char $\mathbb{F}=0$. Let $B=\mathbb{F}[t]$ be the polynomial algebra in the invariable $t, D^{\prime}=\mathbb{F}(d / d t)$. We know that $B\left[D^{\prime}\right] / \mathbb{F}$ is a simple Lie algebra. Let $A=B \times \mathbb{F}=\{(x, a) \mid x \in A, a \in \mathbb{F}\}$ be the associative algebra with the product $(x, a)(y, b)=(x y+b x+a y, a b)$. Then $(0,1)$ is the identity element in $A$. Let $D=\mathbb{F} \partial$, where $\partial((x, a))=\left(\frac{d x}{d t}, 0\right)$. It is not difficult to verify that $\partial$ is a derivation of $A,(B, 0)$ is a $D$-ideal, and $A^{D}=(\mathbb{F}, \mathbb{F})$. So $A$ is not $D$-simple. But we can prove that $A[D] / A^{D} \simeq B\left[D^{\prime}\right] / \mathbb{F}$ is a simple Lie algebra.

Using Theorem 3.9, one can obtain some interesting examples of simple (associative or Lie) algebras of Weyl type which cannot be obtained by the method in SZ1.

Example 3.11. Let char $\mathbb{F}=p>0$, let $A=\mathbb{F}\left[t_{i} \mid 0 \leq i \leq n\right] /\left(t_{i}^{p}-b_{i} \mid 0 \leq i \leq n\right)$ where $0<n<\infty$ and $b_{i} \in \mathbb{F}$, and let $D=\operatorname{span}\left\{\frac{\partial}{\partial t_{i}} \mid 0 \leq i \leq n\right\}$. Then

$$
\begin{aligned}
D(A)=\operatorname{span}\left\{t_{1}^{i_{1}} t_{2}^{i_{2}} \cdots t_{n}^{i_{n}} \mid 0 \leq i_{1}, i_{2}, \ldots, i_{n} \leq p-1,\right. \\
\\
\left.\left(i_{1}, i_{2}, \ldots, i_{n}\right) \neq(p-1, p-1, \ldots, p-1)\right\} .
\end{aligned}
$$

Then $\overline{A[D]}=\left[A[D]^{L}, A[D]^{L}\right] / \mathbb{F}$ is a simple Lie algebra of dimension $p^{2 n}-2$.

Example 3.12. In Example 3.11 if $n=\infty$, then $A[D]^{L} / \mathbb{F}$ is a simple Lie algebra of infinite dimension.

We would like to conclude this paper by several questions.

Questions. 1. Are there any commutative associative algebras $A$ over a field $F$ without identity element such that $A$ is $D$-simple for some commutative subalgebra $D \subset \operatorname{Der}_{\mathbb{F}}(A) ?$

2. If $A[D] / A^{D}$ is a simple Lie algebra, what conditions do $A$ and $D$ satisfy?

3. If the commutative associative algebra $A$ over a field $F$ does not have an identity element, and $A$ is $D$ simple, is $A[D]$ still simple?

\section{REFERENCES}

[DZ1] D. Z. Dokovic and K. Zhao, Derivations, isomorphisms, and second cohomology of generalized Witt algebras, Trans. Amer. Math. Soc. 350 (2) (1998), 643-664. MR 98d:17031

[DZ2] D. Z. Dokovic and K. Zhao, Generalized Cartan type W Lie algebras in characteristic zero, J. Alg. 195 (1997), 170-210. MR 98j:17021

[DZ3] D. Z. Djokovic and K. Zhao, Derivations, isomorphisms, and second cohomology of generalized Block algebras, Alg. Colloq. 3 (3) (1996), 245-272. MR 97g:17020

[H] I. N. Herstein, On the Lie and Jordan rings of a simple associative ring, Amer. J. Math. 77 (1955), 279-285. MR 16:789e

[J1] D. A. Jordan, On the simplicity of Lie algebras of derivations of commutative algebras, J. Alg. 228 (2000), 580-585. MR 2001d:16052

[J2] D. A. Jordan, Iterated skew-polynomial rings and quantum groups, J. Alg. 156 (1993), 194-218. MR 94b:16034

[K] N. Kawamoto, Generalizations of Witt algebras over a field of characteristic zero, Hiroshima Math. J. 16 (1986), 417-462. MR 88d:17017

[O] J. M. Osborn, New simple infinite-dimensional Lie algebras of characteristic 0, J. Alg. 185 (1996), 820-835. MR 98a:17035

[OZ1] J. M. Osborn and K. Zhao, Generalized Poisson bracket and Lie algebras of type $\mathrm{H}$ in characteristic 0, Math. Z. 230 (1999), 107-143. MR 2000c:17038

[OZ2] J. M. Osborn and K. Zhao, Generalized Cartan type K Lie algebras in characteristic 0, Comm. Alg. 25 (1997), 3325-3360. MR 98e:17032 
[P] D. P. Passman, Simple Lie algebras of Witt type, J. Alg. 206 (1998), 682-692. MR 99j:17012

[S1] Y. Su, Derivations of generalized Weyl algebras, Science in China, to appear.

[S2] Y. Su, 2-Cocycles on the Lie algebras of generalized differential operators, Comm. Alg., to appear.

[SZ1] Y. Su and K. Zhao, Simple algebras of Weyl type, Science in China (Series A) 44 (2001), 419-426. CMP 2001:12

[SZ2] Y. Su, K. Zhao, Second cohomology group of generalized Witt type Lie algebras and certain representations, Comm. Alg., to appear.

[SZ3] Y. Su, K. Zhao, Isomorphism classes and automorphism groups of algebras of Weyl type, Science in China (Series A), to appear.

[X] X. Xu, New generalized simple Lie algebras of Cartan type over a field with characteristic 0, J. Alg. 244 (2000), 23-58. MR 2001b:17021

[Z1] K. Zhao, Automorphisms of algebras of differential operators, J. of Capital Normal University 1 (1994), 1-8.

[Z2] K. Zhao, Lie algebras of derivations of algebras of differential operators, Chinese Science Bulletin 38 (10) (1993), 793-798.

[Z3] K. Zhao, Isomorphisms between generalized Cartan type $\mathrm{W}$ Lie algebras in characteristic zero, Canadian J. Math. 50 (1998), 210-224. MR 99e:17035

Institute of Mathematics, Academy of Mathematics and Systems Sciences, Chinese Academy of Sciences, Beijing 100080, People's Republic of China

E-mail address: kzhao@math08.math.ac.cn 